\title{
Filament velocity scaling laws for warm ions
}

\author{
P. Manz ${ }^{1,2}$, D. Carralero², G. Birkenmeier², H.W. Müller², \\ S.H. Müller ${ }^{3}$, G. Fuchert ${ }^{4}$, B.D. Scott $^{2}$, U. Stroth ${ }^{2,1}$ \\ 1 Physik-Department E28, Technische Universität München, \\ James-Franck-Str. 1, 85748 Garching, Germany \\ 2 Max-Planck-Institut für Plasmaphysik, EURATOM Assoziation, \\ Boltzmannstr. 2, 85748 Garching, Germany \\ 3 Center for Momentum Transport and Flow Organization, \\ University of California at San Diego, San Diego, USA \\ 4 Insitut für Grenzflächenverfahrenstechnik und Plasmatechnologie, \\ Universität Stuttgart, Stuttgart, Germany
}

(Dated: August 8, 2013)

\begin{abstract}
The dynamics of filaments or blobs in the scape-off layer of magnetic fusion devices are studied by magnitude estimates of a comprehensive drift-interchange-Alfvén fluid model. The standard blob models are reproduced in the cold ion case. Even though usually neglected, in the scrape-off layer the ion temperature can exceed the electron temperature by an order of magnitude. The ion pressure effects the dynamics of filaments amongst others by adding up to the interchange drive and the polarisation current. It is shown, how both effects modify the scaling laws for filament velocity in dependence of its size. Simplifications for experimental relevant limit regimes are given. These are the sheath dissipation, collisional and electromagnetic regime.
\end{abstract}




\section{INTRODUCTION}

One of the critical issues of magnetically confined fusion devices is the exhaust of particles and heat without seriously damaging the vessel walls of the device. The transport in the region of open field lines beyond the confined region, called scape-off layer (SOL), is dominated by filamentary structures, which are elongated along the magnetic field lines and localized in the drift plane perpendicular to them. This localization gave them the name blobs. Blob motion has been and is extensively studied in fusion devices, but the comparison with theoretical models is constrained by limited diagnostic accessibility. That gave basic low-temperature experiments the possibility to accomplish theory comparisons in more detail, where the satisfying results seem to imply a robust almost understood theoretical description of the plasma blob dynamics. However, most of the blob theories and simulations invoke cold ion models. While $T_{i} \ll T_{e}$ is realistic for most basic plasma physics experiments [1-8], it is not realistic for the tokamak scrape-off layer (SOL), where $T_{i}>T_{e}$ is typical [9]. While theory and experiments of plasma blobs in the cold ion case seem to converge [10], the SOL physics in the warm ion case is not yet understood and investigations are still rare [11-13]. The ion temperature enters the physics in several ways: First, ion pressure adds to the electron pressure of the interchange drive, which is responsible for charge separation, propagation and therefore for the transport capabilities of the blobs. Second, ion temperature effects the Bohm sheath condition and third it leads to polarisation currents and thus adds to the vorticity. Further effects cannot be excluded.

Here, scaling laws for the filament or blob velocity in dependence of its size in the presence of warm ions for the limit cases of the inertial, sheath dissipative, collisional and electromagnetic regime are derived. The scaling between blob velocity $v_{b}$ and size $\delta_{b}$ is one of the most experimentally studied dependences of plasma blobs or filaments and therefore of interest for experimental investigations in fusion experiments. From the blob velocity further information can be obtained. As a first approximation the blob velocity also gives the effective growth rate $\omega_{b} \approx v_{b} / \delta_{b}$ and the scape-off layer width $L_{\perp} \approx v_{b} \tau$ [14], with blob dissipation time scale $\tau$, which is for example $\tau=L_{\|} / 2 c_{s}$ for sheath connected plasmas with connection length $L_{\|}$. 


\section{STANDARD BLOB MODELS}

The standard blob model provides a simple radial transport mechanism. Starting with a monopole (single peaked) density perturbation with a peak value higher than 2.5 times the surrounding rms level, the magnetic curvature induces a charge polarization. Two $E \times B$ flow vortices with different signs are created poloidally above and below the blob. The blob has an associated potential and vorticity dipole structure. The charge separation creates a poloidal electric field, and the resulting $E \times B$ drift moves the density in the direction of lower magnetic field strength or to larger major radius $R$.

The most prominent blob scaling based on the pioneer work of Krasheninnikov addresses its radial propagation given by $[10,15,16]$

$$
v_{b}=2 c_{s}\left(\frac{\rho_{s}}{\delta_{b}}\right)^{2} \frac{L_{\|}}{R},
$$

where $c_{s}$ is the sound speed, $L_{\|}$the parallel connection length, $\rho_{s}=\sqrt{T_{e} m_{i}} / e B$ with electron temperature $T_{e}$, ion mass $m_{i}$, magnetic field strength $B$ and elementary charge $e$. In this model the radial blob velocity scales inversely with the square of the size of the blob $\delta_{b}$. The application of this model to the radial propagation of ELM induced filaments has been developed by Fundamenski [17]. The Krasheninnikov model is derived in the following way from the polarisation equation [18]

$$
\nabla \cdot \frac{d}{d t}\left(e n \rho_{s}^{2} \frac{e}{T_{e}} \nabla_{\perp} \phi\right)+\frac{2 c_{s}^{2} m_{i}}{R B} \frac{\partial n}{\partial y}=\frac{2 n e^{2} c_{s}\left(\phi-\phi_{f l}\right)}{L_{\|} T_{e}}
$$

where $\phi$ is the plasma potential. The first term is the vorticity evolution, the second term is the so-called interchange forcing responsible for the charge separation and the term on the right hand side results from the sheath, where the floating potential $\phi_{f l} \approx 3 T_{e} / e$ is assumed to be constant. As a stationary situation the parallel dynamics, i.e. the parallel current (right hand side of Eq. (2)) is balanced with the interchange forcing (second term on the left hand side of Eq. (2)) to gain an expression of the potential dynamics

$$
\phi=\frac{c_{s} \rho_{s}^{2} B L_{\|}}{R} \frac{1}{n} \frac{\partial n}{\partial y}+\phi_{f l} .
$$

The time derivative of the vorticity (first term on the left hand side of Eq. (2)) is neglected, which leaves, as explained before, the polarisation due to the magnetic field curvature as responsible for the blob propagation. It is assumed that the blob is radially advected with 
the $E \times B$ velocity associated with this potential. As the floating potential is constant it does not have impact on the radial velocity

$$
v_{b}=-\frac{1}{B} \frac{\partial \phi}{\partial y}=-\frac{c_{s} \rho_{s}^{2} L_{\|}}{R} \frac{\partial}{\partial y} \frac{1}{n} \frac{\partial n}{\partial y} .
$$

By making the ansatz of an isolated parabolic in $y$ direction shaped blob propagating in radial $(x)$ direction $n(x, y, t)=n(x)\left(x-v_{b} t\right) e^{-\left(y / \delta_{b}\right)^{2}} \rightarrow \frac{\partial}{\partial y} \frac{1}{n} \frac{\partial n}{\partial y}=-\left(2 / \delta_{b}^{2}\right)$ the prominent blob scaling (1) arises.

As described by Garcia, there are several reasons why the conventional approximation of the sheath current may be insufficient [19]. It does not comply with the observed ballooning and transport driven parallel flows and the assumption implies that it should be collisionless, while SOL plasmas often have significant collisionality. By neglecting the parallel current Garcia et al. obtain a blob propagation scaling independent of the parallel scale length and proportional to the square root of the blob size [19]

$$
v_{b} \sim c_{s} \sqrt{\frac{2 \delta_{b}}{R} \tilde{p}_{e}}
$$

where $\tilde{p}_{e}$ gives the blob pressure amplitude normalized to the background pressure. This scaling results from a balance between the first and the second term on the left hand side of Eq. (2)). Recently this so-called inertial regime (Garcia scaling) and the sheath dissipation regime (Krasheninnikov scaling) have been united by order of magnitude estimates [20] or inclusion of neutral collisions [4].

\section{BLOB MODEL WITH WARM IONS}

\section{DALF model}

The DALF model [21-23] describes drift-Alfvén turbulence in toroidal geometry and therefore also considers interchange and MHD instabilities. It has been used mostly for numerical investigations of turbulence, however here only an analytical treatment is intended. The blob scaling laws will be deduced from the vorticity $\tilde{\Omega}$ equation

$$
\frac{d \tilde{\Omega}}{d t}+\frac{\tau_{i}}{B^{2}}(\nabla \nabla \tilde{\phi}):\left(\nabla \nabla \tilde{p}_{e}\right)=B \nabla_{\|} \frac{\tilde{J}_{\|}}{B}-\left(1+\tau_{i}\right) \mathcal{K}\left(\tilde{p}_{e}\right),
$$

the electron pressure $\tilde{p}_{e}$ evolution

$$
\frac{d \tilde{p}_{e}}{d t}=B \nabla_{\|} \frac{\tilde{J}_{\|}-\tilde{u}_{\|}}{B}+\mathcal{K}\left(\tilde{\phi}-\tilde{p}_{e}\right),
$$


and the parallel current $\tilde{J}_{\|}$equation of the DALF model as given by

$$
\hat{\beta} \frac{\partial \tilde{A}_{\|}}{\partial t}+\mu \frac{d \tilde{J}_{\|}}{d t}=\nabla_{\|}\left(p_{e}+\tilde{p}_{e}-\tilde{\phi}\right)-C \tilde{J}_{\|}
$$

The different terms and parameters will be explained now, more details can be found in Refs. [21-23]. the ion sound speed is given by $c_{s}=\sqrt{T_{e} / m_{i}}$ and does not include the contribution from the ions (this is not accidental, it serve the purpose of normalization), $L_{\|}$ is the parallel connection length, $\rho_{s}=\sqrt{T_{e} m_{i}} / e B$ with electron temperature $T_{e}$, ion mass $m_{i}$, magnetic field strength $B$ and elementary charge $e . d / d t=\partial / \partial t+v_{E \times B} \cdot \nabla$ is the advective derivative with $E \times B$ velocity $v_{E \times B}$. The DALF model also includes an equation for the parallel ion velocity $\tilde{u}_{\|}$including sound-wave physics, which is neglected here by assuming the parallel ion motion to be constant $\tilde{u}_{\|}=c_{s}$. The main fluctuating quantities are the electrostatic potential $\tilde{\phi}=e \phi / T_{e 0}$ normalized to the background mean electron temperature $T_{e 0}$ and the electron pressure fluctuations $\tilde{p}_{e 1}$ normalized to the mean background pressure $p_{e 0}\left(\tilde{p}_{e}=\tilde{p}_{e 1} / p_{e 0}\right)$. Times are normalized to $L_{\perp} / c_{s}$, perpendicular spatial scales to $\rho_{s}$ and parallel length scales are normalized to the parallel connection length $L_{\|} / 2 \pi$, where $L_{\perp}$ is the mean profile scale length. Relative amplitudes of the fluctuations are given in $\rho_{s} / L_{\perp}$. The ratio between ion and electron temperature is given by $\tau_{i}=T_{i} / T_{e}$. The normalized magnetic field strength is $B=1$.

The total ion flow stream function $\tilde{W}=\tilde{\phi}+\tau_{i} \tilde{p}_{e}$ determines the vorticity $\tilde{\Omega}=$ $\left(1 / B^{2}\right) \nabla_{\perp}^{2} \tilde{W}$. The first main difference to the standard blob models will result from the inclusion of the ion diamagnetic contribution $\tilde{p}_{i}=\tau_{i} \tilde{p}_{e}$ to the polarisation drift, which is neglected in the cold ion case. The negligence of $\nabla_{\perp}^{2} \tilde{p}_{i}$ in the vorticity is equal to the negligence of the ion diamagnetic contribution to the polarisation drift, which indirectly assumes MHD ordering [24]. If $\nabla_{\perp}^{2} \tilde{p}_{i}$ is neglected, also $\nabla_{\|} \tilde{J}_{\|}$should be neglected in a self-consistent treatment [24], which has been done in the Garcia scaling (3).

The second term in Eq. (4) is the ion diamagnetic nonlinearity, which cascades energy from larger to smaller scales $[25]$. It can be also written as $\left[\nabla_{\perp} \tilde{\phi}, \nabla_{\perp} \tilde{p}_{i}\right][24]$ consistent with the models in $[11,12]$. Here $[\cdot, \cdot]$ denotes the Poisson bracket. The ion diamagnetic nonlinearity is neglected in the derivation of the scaling laws here and its impact is only touched briefly.

The coordinates used here are $(x, y, s)$, where locally $x$ is radial, $y$ is binormal and $s$ in direction of the unperturbed magnetic field line. The curvature operator is given by 
$\mathcal{K}=\omega_{B}\left(\sin s \partial_{x}+\cos s \partial_{y}\right)$ with $\omega_{B}=2 L_{\perp} / R$ with the curvature radius $R$, which is set to the major radius. Neglecting the geodesic curvature and the magnetic shear the curvature operator reduces to $\mathcal{K}=\omega_{B} \partial_{y}$. Therefore the interchange forcing is given by $\omega_{B}$. The interchange effect provides an effective gravity in the notation of blob theory. The effective gravity is in direct competition with the first term on the right hand side of Eq. (4). Both terms are responsible for the transfer of free to kinetic energy [26]. If the first term dominates we are in the drift-wave regime, which is accompanied by an almost depletion of the density gradients within the blob resulting in more diffuse blobs at greatly reduced velocities [27]. If the effective gravity dominates we are in the interchange regime. In general due to the nonlinear vorticity advection the high wavenumber $k_{\perp} \rho_{s} \geq 0.1$ and corresponding high frequency regimes can be expected to be drift-wave dominated, whereas the low wavenumbers, where plasma blobs are expected, should be dominated by the linear instabilities [26]. The ion to electron temperature ratio $\tau_{i}$ does increase the relative impact of the effective gravity against the parallel divergence of the current and therefore increases the drive of blobs.

The parallel current $\tilde{J}_{\|}=-\nabla_{\perp}^{2} \tilde{A}_{\|}$induces changes in the magnetic field, where $A_{\|}$is the parallel component of the magnetic vector potential. The equations (4) and (6) describe Alfvén dynamics, (4) and (5) drift-wave and interchange dynamics. Different regimes are set by $\hat{\beta}=\left(4 \pi n T_{e} / B^{2}\right)\left(L_{\|} / L_{\perp}\right)^{2}$ and $\hat{\mu}=\left(m_{e} / m_{i}\right)\left(L_{\|} / L_{\perp}\right)^{2}$, which determines the relative transit Alfvén and electron thermal frequencies, respectively. Electromagnetic effects are important, if $\hat{\beta}>\hat{\mu}$ equivalent to $\beta=\hat{\beta}\left(L_{\perp} / L_{\|}\right)^{2}=\left(c_{s} / v_{A}\right)^{2}>\left(m_{e} / m_{i}\right)$ with the Alfvén velocity $v_{A}$. This is called finite $\beta$ turbulence. The collisionality is given by $C=\nu \hat{\mu}$ with $\nu=\nu_{e i}\left(L_{\perp} / c_{s}\right)$, where $\nu_{e i}$ is the ion-electron collision frequency.

To get a feeling for these three dimensionless parameters $(\hat{\beta}, \hat{\mu}, C)$ at different radial positions (separatrix, near SOL, far SOL) results from a multi-machine comparison [28] are briefly summarized now. In $H$ mode at the separatrix $\hat{\beta} \sim 10^{2}-10^{4}$ and $\hat{\mu} \sim 10^{2}-10^{4}$, at the near SOL $\hat{\beta} \sim 10^{1-10^{2}}$ and $\hat{\mu} \sim 10^{2}-10^{4}$, while in the far SOL $\hat{\beta} \sim 10^{-2}-1$ and $\hat{\mu} \sim 10^{2}-10^{4}$. The collisonality is between 10 and $10^{5}$. In $L$ mode at the separatrix $\hat{\beta} \sim 10^{1}$ and $\hat{\mu} \sim 1-10^{2}$, at the near SOL $\hat{\beta} \sim 10^{1}$ and $\hat{\mu} \sim 10^{2}$, while in the far SOL $\hat{\beta} \sim 10^{-2}-10^{-1}$ and $\hat{\mu} \sim 1-10^{2}$. The collisonality is between 1 and $10^{2}$.

To investigate the impact of the ion dynamics on the blob dynamics the parallel current equation (6) will be further simplified. First, no variation of the background pressure on a 
field line $\nabla_{\|} p_{e}=0$ is assumed. Second, the normalized mass ratio $\hat{\mu}$ is neglected, which is justified as long as the effective growth rate of the blob stays below the ion-electron collision frequency. Both contributions to Eq. (6) possibly have an impact on the blob dynamics, but neither they result from the ion dynamics nor they are considered in the standard blob models. We leave these effects for future investigations.

In summary, the ion to electron temperature ratio $\tau_{i}$ has three main effects, all in the vorticity equation: It increases the interchange forcing and the drive of the blobs, it leads to polarisation currents which have a direct impact on the vorticity and it induces an additional nonlinearity which cascades energy to smaller scales, which can break blobs apart.

\section{Blob model with warm ions and the hierarchy of scalings}

Due to the simplifications explained above the scaling laws will be derived from the evolution of the polarisation

$$
\frac{d \nabla_{\perp}^{2}\left(\tilde{\phi}+\tau_{i} \tilde{p}_{e}\right)}{d t}=\nabla_{\|} \tilde{J}_{\|}-\left(1+\tau_{i}\right) \frac{2 L_{\perp}}{R} \frac{\partial}{\partial y} \tilde{p}_{e}
$$

the electron pressure

$$
\frac{d \tilde{p}_{e}}{d t}=\nabla_{\|} \tilde{J}_{\|}+\frac{2 L_{\perp}}{R} \frac{\partial}{\partial y}\left(\tilde{\phi}-\tilde{p}_{e}\right)
$$

and the parallel current

$$
\beta \frac{\partial \tilde{A}_{\|}}{\partial t}=\nabla_{\|}\left(\tilde{p}_{e}-\tilde{\phi}\right)-C \tilde{J}_{\|} \cdot
$$

With the blob correspondence principle $[10,18]$ the linear instability of these fluid equations is related to the radial blob velocity and scale size by

$$
\begin{aligned}
& \omega_{b} \rightarrow \frac{v_{b}}{\delta_{b}} \\
& k_{\perp} \rightarrow \frac{1}{\delta_{b}},
\end{aligned}
$$

where $\omega_{b}$ is the characteristic blob frequency and $k_{\perp}$ is its perpendicular wavenumber. In the inertial regime of the standard blob models this characteristic blob frequency is a growth rate $\gamma_{b}$. In principle the blob correspondence principle also includes $L_{\perp} \rightarrow \delta_{b}$ and $k_{\|} \rightarrow 1 / L_{\|}$, which is not applied here. The blob velocity is determined by the polarisation (7), where in the inertial (or resistive) regime $\nabla_{\|} \tilde{J}_{\|}=0$. If losses to the wall become important the sheath dissipation has to be considered. Sheath dissipation $\sigma \phi / B[20]$ with sheath conductivity 
$\sigma=c_{s} / L_{\|} \rho_{s}^{2}$ can be included in the polarisation equation (7). Due to its physical dimension it has to be normalized by $L_{\perp} \rho_{s}^{2} / c_{s}$ and it is included as $\sigma=L_{\perp} / L_{\|}$resulting in

$$
\nabla_{\|} \tilde{J}_{\|}=L_{\perp} / L_{\|} \tilde{\phi}
$$

In the collisional regime the parallel current will be determined by Eq. (9) (in the limit of high collisonality). Both regimes contain the inertial regime as a limiting case. To decide in which regime the experiments are done, one has to compare the sheath resistivity $\eta_{s h}=L_{\|} / L_{\perp}$ with the collisional resistivity $\eta_{c}=C$ (derived from Eq. (9) neglecting electromagnetic effects and assuming, that potential and pressure fluctuations are phase shifted by $\pi / 2$ for interchange modes). For $\Lambda=\left(\nu_{e i} L_{\|}\right) /\left(\omega_{c e} \rho_{s}\right)=C\left(L_{\perp} / L_{\|}\right)<1$ the sheath is dominating the parallel resistivity for $\Lambda>1$ the collisonality is the relevant dissipation mechanism. Here $\omega_{c e}$ is the electron cyclotron frequency. The boundary between sheath dissipation and collisional regime at $\Lambda=1$ has been also found in Ref. [29]. In particular interesting for ELM filaments are large-scale events, which are in the electromagnetic regime if $\left(v_{b} / c_{s}\right)\left(\delta_{b} / \rho_{s}\right)\left(L_{\perp} / \rho_{s}\right)>(C / \hat{\beta})$ (balancing electromagnetic and collisional effects in Eq. (9) under the blob corresponding principle). In the electromagnetic regime, additionally, Eq. (8) will be used to determine the adiabatic coupling.

\section{LIMITING CASES}

\section{Inertial regime}

In the limit of a highly resistive plasma the parallel current is neglected. This regime corresponds to the hydrodynamic regime in the Hasegawa-Wakatani frame [30]. First as consistency check, the standard cold ion case $\left(\tau_{i}=0\right)$ (the case under MHD ordering) is derived. Than the warm ion case $\left(\tau_{i} \gg 1\right)$ is studied and finally both regimes are united.

In the cold ion case, both the polarisation velocity and the vorticity are given by the potential $W=\tilde{\phi}$. The renormalized polarisation equation (7) then reads

$$
\frac{L_{\perp}}{c_{s}} \frac{d}{d t} \rho_{s}^{2} \nabla_{\perp}^{2} \frac{L_{\perp}}{\rho_{s}} \tilde{\phi}=-\frac{2 L_{\perp}}{R} \rho_{s} \frac{\partial}{\partial y} \frac{L_{\perp}}{\rho_{s}} \tilde{p_{e}} .
$$

The blob velocity is the $E \times B$ velocity $\nabla \tilde{\phi}=-v_{b} /\left(\rho_{s} c_{s}\right)$

$$
-\frac{L_{\perp}}{c_{s}^{2}} \frac{d}{d t} \rho_{s} \nabla_{\perp} \frac{L_{\perp}}{\rho_{s}} v_{b}=-\frac{2 L_{\perp}}{R} \rho_{s} \frac{\partial}{\partial y} \frac{L_{\perp}}{\rho_{s}} \tilde{p}_{e}
$$


Applying dimensional analysis $\left(\partial / \partial x \rightarrow i k_{x}, \partial / \partial y \rightarrow i k_{y}\right)$ to Eq. (14), assuming the blob is purely growing $\left(/ \mathrm{t} \rightarrow \gamma_{b}\right.$ the blob correspondence principle $\left(\gamma_{b}=v_{b} / \delta_{b}, \delta_{b}=1 / k\right)$ we obtain

$$
\frac{L_{\perp}^{2}}{c_{s}^{2}} \frac{v_{b}^{2}}{\delta_{b}} \frac{1}{\delta_{b}}=\frac{2 L_{\perp}}{R} \rho_{s} \frac{1}{\delta_{b}} \frac{L_{\perp}}{\rho_{s}} \tilde{p_{e}}
$$

and recover the scaling of Garcia (3). We want to note that all derivations in this contribution are also valid for blobs, that are more radially than poloidally extended $\delta_{b}=1 / k_{y} \gg 1 / k_{x}$.

For moderate to high $\tau_{i}$ and/or strong pressure fluctuations which seems certainly justified for a blob, the ion flow stream function is determined by the ion pressure contribution $W=\phi+\tau_{i} p_{e} \approx \tau_{i} p_{e}$ and the polarisation equation (7) reads

$$
\frac{L_{\perp}}{c_{s}} \frac{d}{d t} \rho_{s}^{2} \nabla_{\perp}^{2} \frac{L_{\perp}}{\rho_{s}} \tau_{i} \tilde{p}_{e}=-\left(1+\tau_{i}\right) \frac{2 L_{\perp}}{R} \rho_{s} \frac{\partial}{\partial y} \frac{L_{\perp}}{\rho_{s}} \tilde{p}_{e}
$$

Applying dimensional analysis $\left(d / d t \rightarrow i \omega_{b}=i v_{b} / \delta_{b}\right)$

$$
-\frac{L_{\perp}}{c_{s}} \frac{v_{b}}{\delta_{b}} \rho_{s}^{2} \frac{1}{\delta_{b}^{2}} \frac{L_{\perp}}{\rho_{s}} \tau_{i} \tilde{p_{e}}=-\left(1+\tau_{i}\right) \frac{2 L_{\perp}}{R} \rho_{s} \frac{1}{\delta_{b}} \frac{L_{\perp}}{\rho_{s}} \tilde{p}_{e} .
$$

From the equation above a scaling law for blob propagation can be derived

$$
v_{b}=2 c_{s} \frac{\left(1+\tau_{i}\right)}{\tau_{i}}\left(\frac{\delta_{b}}{\rho_{s}}\right)^{2} \frac{\rho_{s}}{R}
$$

which scales with the square of the blob size in difference to the standard models in Eqs. (1) and (3).

Before we take both contributions to the vorticity into account, we want to discuss the difference of the cold and warm ion cases in more detail. As the electrostatic potential $\phi$ has been neglected in the total ion stream function $W$, one might think that the electrostatic potential is not important for the blob dynamics and may ask the question, what is the mechanism for blob propagation? The charge separation responsible for the blob motion is hidden in the term $d / d t=\partial / \partial t+v_{x} \partial / \partial x$ with the advection $v_{x}$. Due to the gyroviscous cancellation upon advection the advective derivative $v_{x}$ is given by the $E \times B$ velocity only $[26,31]$. As $i \omega_{b}=d / d t \approx v_{x} \partial / \partial x \approx i v_{b} / \delta_{b}$ the advective motion due to charge separation is contained in $v_{b}=\omega_{b} \delta_{b}$. The propagation is still given by the $E \times B$ velocity due to the charge separation resulting from the interchange drive. This can be also interpreted as wave propagation $v_{b}=\omega / k_{x}=\omega_{b} \delta_{b}$. In the cold ion case the blob correspondence principle is used to relate the blob growth rate to the blob velocity and size by dimensional arguments 
$d / d t \approx \partial / \partial t=\gamma_{b}=v_{b} / \delta_{b}$, in the warm ion case it is an advection induced dispersion $d / d t \approx v_{x} \partial / \partial x \approx i v_{b} / \delta_{b}=i \omega_{b}$ balancing the effective gravity in stationary conditions.

An important difference to the cold ion case is that the dipolar vortex induced by the interchange drive is due to the ion pressure. This dipolar perturbation adds to the blob pressure inducing an asymmetry in poloidal direction, a feature also seen in simulations $[11,13]$. Due to the asymmetry additional dipoles may form [13]. As the interchange drive will be stronger at the steeper flank this part of the blob will move faster resulting in a tilt of the blob. Due to this tilt the blob velocity is no longer just in the radial direction and a large fraction of the blob velocity $v_{b}$ given by Eq. (18) may be in the poloidal direction. Also this has been seen in simulations $[11,13]$. The poloidal fraction of the blob velocity will increase with the distance to the separatrix, effectively reducing the radial velocity of the blob.

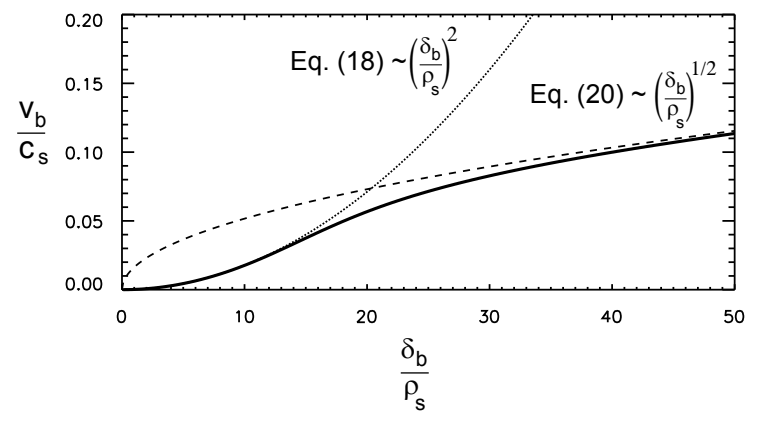

FIG. 1: Blob velocity against blob size in the hot ion inertial regime. Plasma parameters used for this example are $\tau_{i}=3, \tilde{p}_{e}=1, \rho_{s}=10^{-4} \mathrm{~m}, R=1.5 \mathrm{~m}$. Blobs with $\delta_{b} / \rho_{s} \ll$ $\sqrt[3]{\tau_{i}^{2} R /\left(8\left(1+\tau_{i}\right) \rho_{s}\right)} \approx 16$ are dominated by the ion contribution to the polarization, larger blobs are determined by the plasma potential.

Finally both contributions $\nabla_{\perp}^{2} \tilde{\phi}$ and $\nabla_{\perp}^{2} \tilde{p}_{i}$ to the vorticity are taken into account. We apply $d / d t \rightarrow i \omega_{b}$ to both terms. The contributions from potential and pressure fluctuations are not in phase obvious from the imaginary parts

$$
\left(\frac{v_{b}}{c_{s}}\right)^{2} \frac{1}{\delta_{b}^{2}}-i\left(\frac{v_{b}}{c_{s}}\right) \frac{\tau_{i} \rho_{s}}{\delta_{b}^{3}} \tilde{p}_{e}=-i\left(1+\tau_{i}\right) \frac{2}{R \delta_{b}} \tilde{p}_{e} .
$$

After completing the square

$$
\left(\left(\frac{v_{b}}{c_{s}}\right)-i\left(\frac{\tau_{i} \rho_{s}}{2 \delta_{b}} \tilde{p}_{e}\right)\right)^{2}=-\underbrace{\left(\frac{\tau_{i} \rho_{s}}{2 \delta_{b}} \tilde{p}_{e}\right)^{2}}_{f_{i}}-i \underbrace{\left(1+\tau_{i}\right) \frac{2 \delta_{b}}{R} \tilde{p}_{e}}_{g}
$$


and matching real and imaginary parts, the blob velocity is given by

$$
\left|\frac{v_{b}}{c_{s}}\right|=\sqrt{\frac{\sqrt{f_{i}^{2}+g^{2}}-f_{i}}{2}} .
$$

This result has two limits illustrated by Fig. 1. For $|g| \gg\left|f_{i}\right|$, which holds for large blobs $\left(\delta_{b} / \rho_{s}\right)^{3} \gg \tau_{i}^{2} R \tilde{p}_{e} /\left(8\left(1+\tau_{i}\right) \rho_{s}\right)$, besides a factor of $\sqrt{\left(1+\tau_{i}\right) / 2}$, the $\tau_{i}$-modified Garcia scaling is obtained

$$
\left|\frac{v_{b}}{c_{s}}\right|=\sqrt{\left(1+\tau_{i}\right) \frac{\delta_{b}}{R} \tilde{p}_{e}} .
$$

For smaller blobs $|g| \ll\left|f_{i}\right|$ and since $f_{i}>0$

$$
\begin{array}{r}
\left|\frac{v_{b}}{c_{s}}\right|=\sqrt{\frac{f_{i} \sqrt{1+g^{2} / f_{i}^{2}}-f_{i}}{2}} \\
\approx \sqrt{\frac{f_{i}\left(1+g^{2} / 2 f_{i}^{2}\right)-f_{i}}{2}}=\frac{1}{2} \sqrt{\frac{g^{2}}{f_{i}}}
\end{array}
$$

Eq. (18) is recovered. Therefore there are two inertial regimes, for smaller blobs the ion diamagnetic contribution to the polarisation current is responsible for their acceleration, where for larger blobs it is the $E \times B$ contribution. We call the first one the ion pressure dominated resistive ballooning regime (iRB) and the second the conventional resistive ballooning regime $(\mathrm{RB})$. For typical SOL parameters $\left(\rho_{s} \sim 10^{-4} \mathrm{~m}, R \sim 1 \mathrm{~m}\right)$ the boundary between quadratic and square root blob size dependence is about $\delta_{b} / \rho_{s} \sim 10$.

The ion diamagnetic nonlinearity $\frac{\tau_{i}}{B^{2}}(\nabla \nabla \tilde{\phi}):\left(\nabla \nabla \tilde{p}_{e}\right)$ is in the frame of the blob correspondence principle equal to the left-hand side of Eq. (16). Therefore Eq. (18) gives also the maximum radial velocity at a given size in the linear approximation. Faster or non-isotropic structures will be strongly effected by the direct cascade of the ion diamagnetic nonlinearity and those structures will decay. Because the ion diamagnetic nonlinearity is dimensionally equal to the ion diamagnetic contribution to the polarisation one might expect that those cancel each other leaving the same equation as in the cold ion case. Therefore even if the physics is much more complicated as in the derivation of the Garcia scaling (3) it still could be valid in the warm ion case. Because potential and pressure perturbations do not have the same spatial structure and the ion diamagnetic nonlinearity influence is stronger on smaller structures compared to the ion diamagnetic contribution to the polarisation, just dimensional arguments could be misleading. A detailed numerical study is necessary to investigate the canceling capabilities of the ion diamagnetic nonlinearity and its effects on the blob dynamics, which we leave for future investigations. 


\section{Sheath dissipation regime}

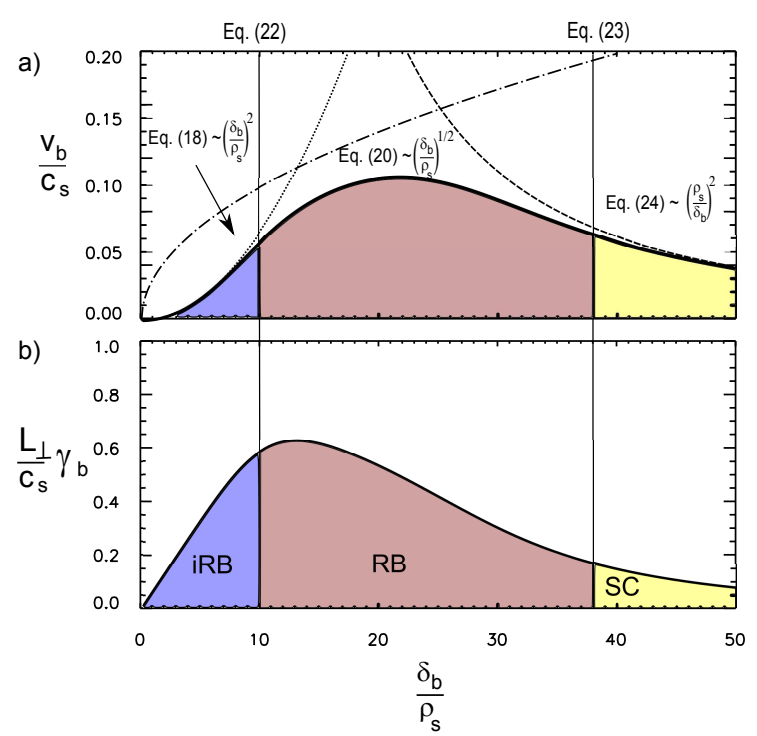

FIG. 2: Blob velocity (a) and effective growth rate (b) against blob size in the sheath dissipation regime. Plasma parameters used for this example are $\tau_{i}=3, \tilde{p}_{e}=1, \rho_{s}=10^{-4} \mathrm{~m}$, $R=0.4 \mathrm{~m}, L_{\|}=10 \mathrm{~m}$. Blobs with $\delta_{b} / \rho_{s} \ll \sqrt[3]{\tau_{i}^{2} R /\left(8\left(1+\tau_{i}\right) \rho_{s}\right)} \approx 10$ are dominated by the ion contribution to the polarization, larger blobs are determined by the plasma potential. Above $\left(\delta_{b} / \rho_{s}\right) \approx \sqrt[5]{8\left(1+\tau_{i}\right) \tilde{p}_{e} L_{\|}^{2} /\left(\rho_{s} R\right)} \approx 38$ the sheath dissipation dominates.

To model losses to the wall the divergence in the parallel current $\nabla_{\|} \tilde{J}_{\|}=L_{\perp} / L_{\|} \phi$ is taken into account. Just this term is included in the polarisation equation (7), everything else stays the same as in the full model Eq. (19). The sheath dissipation adds to the seconds term in Eq. (19) with $i\left(1 / L_{\|}\right)\left(\delta_{b}^{3} / \rho_{s}^{2}\right)\left(v_{b} / c_{s}\right)$. The balance between the seconds term in Eq. (19) and $i\left(1 / L_{\|}\right)\left(\delta_{b}^{3} / \rho_{s}^{2}\right)\left(v_{b} / c_{s}\right)$ gives the boundary

$$
\left(\delta_{b} / \rho_{s}\right)=\sqrt[4]{\tau_{i}\left(L_{\|} / \rho_{s}\right) \tilde{p}_{e}}
$$

between the inertial and the sheath effected regime. Blobs smaller than $\left(\delta_{b} / \rho_{s}\right)^{4}<$ $\tau_{i}\left(L_{\|} / \rho_{s}\right) \tilde{p}_{e}$ are in the iRB inertial regime (18) (Fig. 2). If the sheath dissipation is stronger than the ion diamagnetic contribution to the polarisation, $f_{i}$ can be substituted by $f_{c}=\left(\left(1 / L_{\|}\right)\left(\delta_{b}^{3} / \rho_{s}^{2}\right)\right)^{2}$. There are two subregimes of the sheath effected regime. The boundary is given by $|g|=\left|f_{c}\right|$ or

$$
\left(\delta_{b} / \rho_{s}\right) \approx \sqrt[5]{8\left(1+\tau_{i}\right) \tilde{p}_{e} L_{\|}^{2} /\left(\rho_{s} R\right)} .
$$


For blobs smaller than $\left(\delta_{b} / \rho_{s}\right)^{5} \ll 8\left(1+\tau_{i}\right) \tilde{p}_{e} L_{\|}^{2} / \rho_{s} R$ the interchange forcing is most effective $\left(|g| \gg\left|f_{c}\right|\right)$ and the inertial RB scaling (20) recovers. As seen in Fig. 2 the RB scaling gives an inaccurate reproduction of the curve taking all terms into account. It is advised to take all terms into account in this regime. Further, it should be noted that the difference between the first and the second boundary is usually small, therefore the RB regime is very narrow and usually more difficult to see than in Fig. 2. Larger blobs will follow the the Krasheninnikov scaling

$$
\left|\frac{v_{b}}{c_{s}}\right|=\left(1+\tau_{i}\right)\left(\frac{L_{\|}}{R}\right)\left(\frac{\rho_{s}}{\delta_{b}}\right)^{2} \tilde{p}_{e},
$$

beside the additional drive by the factor of $\left(1+\tau_{i}\right) / 2$. This regime will be called the sheath connected (SC) regime, here. Therefore under consideration of sheath dissipation we observe three regimes: the sheath non-effected iRB (18), the interchange dominated conventional $\mathrm{RB}$ regime (20) and the sheath connected regime (24).

Obviously one might ask the question in which of these regime most of the blobs can be expected to occur? The blob correspondence principle states that the effective growth rate relates to the blob velocity by $\gamma_{b}=v_{b} / \delta_{b}$. Due to the additional factor of $1 / \delta_{b}$, the maximum of the effective growth rate exhibits a maximum at the first boundary (22). For larger blobs the growth rate slightly decreases until the second boundary (23), where the growth rate strongly drops to $\sim \delta_{b}^{-3}$. As explained above the diamagnetic nonlinearity, which breaks the blob in smaller structures apart, as well as the tilt by the generation of additional dipoles in the vorticity will reduce the effective growth rate close to the first boundary and shift the scale, where most of the blobs will be detected closer to the second one. Beside a factor of $\sqrt[5]{8\left(1+\tau_{i}\right)} \approx 2$ this expectation is in line with the standard blob theory [18]. As explained above the region of the $\mathrm{RB}$ regime is quite narrow and this might explain, why in experimental investigations often only one blob size is detected [32].

\section{Collisional regime}

Collisional dissipation should be considered for $\Lambda=C\left(L_{\perp} / L_{\|}\right)>1$ instead of the sheath dissipation. In the collisional regime Eq. (9) can be approximated by

$$
\nabla_{\|} \tilde{J}_{\|}=\frac{1}{C} \nabla_{\|}^{2}\left(\tilde{p}_{e}-\tilde{\phi}\right)
$$


instead of Eq. (12). This approximation is also used in the derivation of the HasegawaWakatani equations. The radial velocity is given by the $E \times B$ velocity and $\tilde{\phi}=-i \frac{\delta_{b} v_{b}}{\rho_{s} c_{s}}$. Equation (25) then reads

$$
\nabla_{\|} \tilde{J}_{\|}=-\frac{1}{C}\left(\frac{L_{\|}}{\delta_{\|}}\right)^{2} \frac{L_{\perp}}{\rho_{s}}\left(\tilde{p}_{e}+i \frac{\delta_{b} v_{b}}{\rho_{s} c_{s}}\right) .
$$

Inserting the divergence of the parallel current in Eq. (7), completing the square, matching real and imaginary parts results in

$$
\begin{aligned}
& \left(\left(\frac{v_{b}}{c_{s}}\right)-\frac{1}{2}\left(\frac{\tau_{i} \rho_{s}}{\delta_{b}} \tilde{p}_{e}+\frac{1}{C}\left(\frac{L_{\|}}{\delta_{\|}}\right)^{2} \frac{\delta_{b}^{3}}{\rho_{s}^{2} L_{\perp}}\right) i\right)^{2} \\
& =-\underbrace{\left(1+\tau_{i}\right) \frac{2 \delta_{b}}{R} \tilde{p}_{e}}_{g} i \\
& -\underbrace{\left(\frac{1}{4}\left(\frac{\tau_{i} \rho_{s}}{\delta_{b}} \tilde{p}_{e}+\frac{1}{C}\left(\frac{L_{\|}}{\delta_{\|}}\right)^{2} \frac{\delta_{b}^{3}}{\rho_{s}^{2} L_{\perp}}\right)^{2}-\frac{1}{C}\left(\frac{L_{\|}}{\delta_{\|}}\right)^{2} \frac{\delta_{b}^{2}}{\rho_{s} L_{\perp}} \tilde{p}_{e}\right)}_{f_{c}}
\end{aligned}
$$

As in the sheath dissipative regime the collisionality does only impact the dynamics for blobs larger than the boundary

$$
\left(\delta_{b} / \rho_{s}\right)=\sqrt[4]{\tau_{i}\left(C\left(\delta_{\|} / L_{\|}\right)^{2} L_{\perp} / \rho_{s}\right) \tilde{p}_{e}}
$$

below which blobs are in the $\mathrm{iRB}$ regime. As in the sheath dissipation case there is an intermediate scale, which is dominated by the interchange forcing. The boundary between square root (RB regime) and inverse square dependence is located at

$$
\left(\delta_{b} / \rho_{s}\right) \approx \sqrt[5]{8 C^{2}\left(\delta_{\|} / L_{\|}\right)^{4}\left(1+\tau_{i}\right) \tilde{p}_{e} L_{\perp}^{2} /\left(\rho_{s} R\right)}
$$

Blobs larger than this limit, are in the collisional dissipative regime (col), where they fulfill the scaling

$$
\left|\frac{v_{b}}{c_{s}}\right|=\left(1+\tau_{i}\right) C\left(\frac{\delta_{\|}}{L_{\|}}\right)^{2}\left(\frac{L_{\perp}}{R}\right)\left(\frac{\rho_{s}}{\delta_{b}}\right)^{2} \tilde{p}_{e} .
$$

Therefore the blob velocity increases linear with the collisionality and plasma density $C \sim n$ consistent with experimental observations [32].

\section{Electromagnetic regime}

It is known that ELM filaments carry a substantial current [33]. Due to Ampére's law the parallel current induces a magnetic field $\tilde{J}_{\|}=-\nabla_{\perp}^{2} A_{\|}$, which translates within our scaling 
to $\tilde{A}_{\|}=\left(\delta_{b} / \rho_{s}\right)^{2} \tilde{J}_{\|}$. For large blobs this will give a large contribution even at rather small $\hat{\beta}$. If the electromagnetic contribution dominates over the collisionality, Eq. (9) is given by

$$
\hat{\beta} \frac{L_{\perp}}{c_{s}} \frac{v_{b}}{\delta_{b}}\left(\frac{\delta_{b}}{\rho_{s}}\right)^{2} \tilde{J}_{\|}=-\frac{L_{\perp}}{\rho_{s}} \frac{L_{\|}}{\delta_{\|}}\left(\tilde{\phi}-\tilde{p}_{e}\right)
$$

which is solved for $\left(\tilde{\phi}-\tilde{p}_{e}\right)$ and inserted into (8). From that a response of the parallel current can be deduced

$$
\nabla_{\|} \tilde{J}_{\|}=\frac{\frac{L_{\perp}^{2}}{\rho_{s} \delta_{b}} \frac{v_{b}}{c_{s}} \tilde{p}_{e}}{1-\frac{2 L_{\perp}^{2} \hat{\beta}}{R \rho_{s}}\left(\frac{\delta_{\|}}{L_{\|}}\right)^{2} \frac{v_{b}}{c_{s}}}
$$

For $1 \gg \frac{2 L_{\perp}^{2} \hat{\beta}}{R \rho_{s}}\left(\frac{\delta_{\|}}{L_{\|}}\right)^{2} \frac{v_{b}}{c_{s}}$ the adiabatic limit $\left(\nabla_{\|} \tilde{J}=d / d t\left(\tilde{p}_{e}\right) \rightarrow \tilde{\phi}=\tilde{p}_{e}\right)$. is taken. The electro magnetic effects dominate the divergence of the parallel current for

$$
1 \ll \frac{2 L_{\perp}^{2} \hat{\beta}}{R \rho_{s}}\left(\frac{\delta_{\|}}{L_{\|}}\right)^{2} \frac{v_{b}}{c_{s}} .
$$

Note that here $\hat{\beta}$ is additionally normalized with $\left(L_{\|} / L_{\perp}\right)^{2}$. For example, an experimental observed $\beta=n T /\left(B^{2} / 2 \hat{\mu}_{0}\right)$ in the order of 0.01 translates with $\left(L_{\|} / L_{\perp}\right) \sim 10^{2}$ to a $\hat{\beta}$ in the order of $10^{2}$. Of course, for typical SOL conditions a $\beta$ of only $10^{-5}$ to $10^{-4}$ can be expected, which let basically exclude electromagnetic effects to modify the dynamics. However, at least during pellet ablation, the formation of high $\beta \sim 0.05$ drifting plasmoids has been observed [34] and it can be expected that the local $\beta$ of ELM filaments is much higher than $10^{-4}$. Then Eq. (30) reads

$$
\nabla_{\|} \tilde{J}_{\|}=-\frac{R}{2 \delta_{b} \hat{\beta}}\left(\frac{L_{\|}}{\delta_{\|}}\right)^{2} \tilde{p}_{e} .
$$

This gives the closure for the parallel current in (7). This term adds to the effective gravity. With the Alfvén velocity $v_{A}=B / \sqrt{4 \pi n_{0} m_{i}}, \hat{\beta}=\left(c_{s} / v_{A}\right)^{2}\left(L_{\|} / L_{\perp}\right)^{2}$ and a normalized Alfv́en frequency $\omega_{A}=\left(v_{A} / \delta_{\|}\right)\left(L_{\perp} / c_{s}\right)$ the effective gravity can be written as

$$
g=\omega_{B} \frac{\rho_{s}}{L_{\perp}}\left(1+\tau_{i}+\left(\frac{\omega_{A}}{\omega_{B}}\right)^{2}\right) .
$$

Usually $g \gg f_{i}$ and the radial velocity is given by

$$
\left|\frac{v_{b}}{c_{s}}\right|=\sqrt{\frac{\omega_{B}}{2} \frac{\rho_{s}}{L_{\perp}}\left(1+\tau_{i}+\frac{\omega_{A}^{2}}{\omega_{B}^{2}}\right)\left(\frac{\delta_{b}}{\rho_{s}}\right)}
$$

which gives a square root dependence on the filament size as suggested in experiments [35]. As the contribution of the electromagnetic part $\omega_{A} \sim 1 / \delta_{\|}$depends on the parallel extent of 
the structure, different parallel extents will result in a branch-like distribution in the $v_{b}-\delta_{b}$ scatter plot as experimentally observed [35].

Finally the requirements for the electromagnetic regime are summarized. For blobs larger $\delta_{b} / \rho_{s}>2 \Lambda L_{\|} / R=C \omega_{B}$, also $\left(\omega_{A}^{2} / \omega_{B}\right)\left(\delta_{b} / \rho_{s}\right)>C / \hat{\beta}$ holds. Together with relation (31), which is equal to $\left(L_{\perp} / \rho_{s}\right)\left(v_{b} / c_{s}\right) \gg\left(\omega_{A}^{2} / \omega_{B}\right),\left(v_{b} / c_{s}\right)\left(\delta_{b} / \rho_{s}\right)\left(L_{\perp} / \rho_{s}\right)>(C / \hat{\beta})$ is fulfilled. Therefore, the electromagnetic regime is valid in finite beta turbulence $\left(\beta>m_{e} / m_{i}\right)$, for blobs exceeding the velocity of $\left(v_{b} / c_{s}\right)>\left(R \rho_{s}\right) /\left(2 \beta L_{\|}^{2}\right)$ with a size above $\left(\delta_{b} / \rho_{s}\right)>2 \Lambda L_{\|} / R$.

\section{CONCLUSION}

In the scrape-off layer of fusion experiments the ion temperature often exceeds the electron temperature, which has strong effects on the dynamics of turbulent structures. However, most of blob theory is done for cold ions. Hot ions modify the SOL by strengthening the interchange drive. Besides that, the ion diamagnetic contribution to the polarisation drift can dominate the total ion stream function, which strongly modifies the response of the polarisation current to the interchange drive modifying the scaling between radial velocity and blob size.

By inclusion of the sheath conductivity into the DALF model it has been possible to unite the inertial and different dissipative regimes of blob models under a comprehensive driftinterchange-Alfvén fluid model. Considering warm ions, three regimes are derived, two of them inertial and one dissipative. For blobs smaller than $\approx 10 \rho_{s}$ the blob velocity increases with the square of the blob size (see Eq. (18)). For larger blobs $\left(>10 \rho_{s}\right)$ the usual cold ion inertial scaling (see Eq. (20)) is valid with an additional factor of $\left(1+\tau_{i}\right) / 2$, where $\tau_{i}$ gives the ion to electron temperature ratio.

The velocities of the largest blobs are determined by the parallel dynamics carried by the electrons, which is not modified by the ion temperature. Therefore also the parallel closure and the classification in different limit regimes is not effected. In the electrostatic case, the distinction between sheath limited and collisional dissipative regimes is set by $\Lambda=C\left(L_{\perp} / L_{\|}\right)$as in the cold ion case. The results are summerized in Fig. 3. In both cases two inertial and one specific dissipative regime are observed. As in the cold ion case in the sheath dissipative or collisional regime the blob velocity increases linear with the parallel connection length (see Eq. (24)) or collisionality (see Eq. (28)), respectively. Also here we 


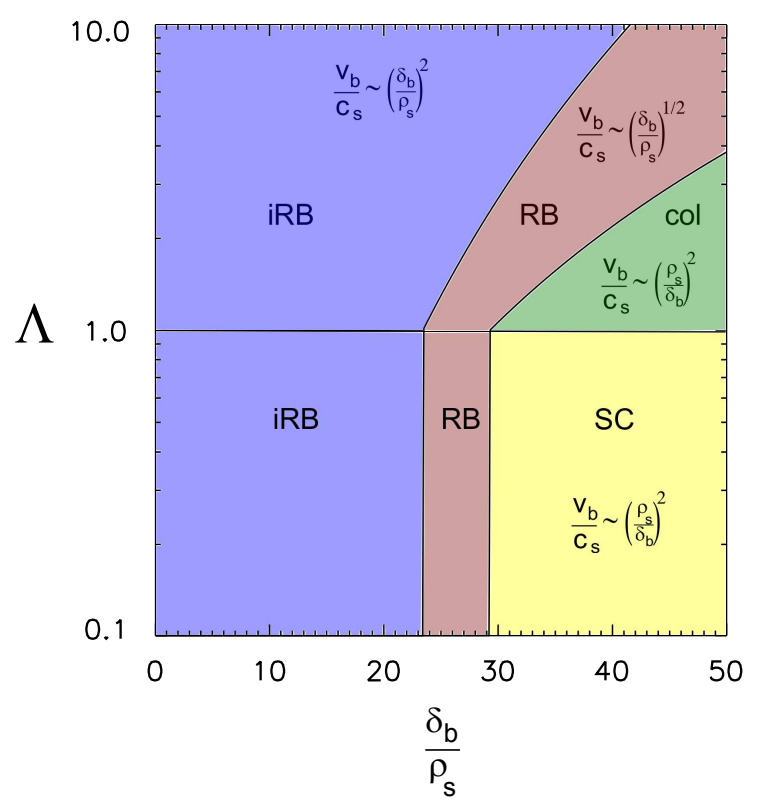

FIG. 3: Blob regimes for different collisionalities $\Lambda$ and scales $\delta_{b}$. The inertial regime exhibits two scaling regimes, the conventional resistive ballooning (RB) regime described by Eq. (20) and the ion pressure dominated resistive ballooning (iRB) regime, where the blob velocity-size scaling is given by Eq. (18). Larger blobs undergo dissipative effects, depending on the collisionality $\Lambda$. For $\Lambda<1$ the sheath dissipation dominates (sheath connected (SC) regime Eq. (24)), for $\Lambda>1$ the blob is in the collisional (col) regime Eq. (28). Plasma parameters used for this example are $\tau_{i}=3$, $\tilde{p}_{e}=1, \rho_{s}=10^{-4} \mathrm{~m}, R=1.5 \mathrm{~m}, L_{\|}=10 \mathrm{~m}$.

find an additional factor of $\left(1+\tau_{i}\right) / 2$ for finite $\tau_{i}$. Also the blob size scales similar to the cold ion case with an additional factor of about $\sqrt[5]{8\left(1+\tau_{i}\right)} \approx 2$ (between 1.7 for $\tau_{i}=1$ and 2.4 for $\left.\tau_{i}=10\right)$.

The DALF model allows also to study electromagnetic effects. These are possibly relevant for ELMs. In the finite beta regime $\left(\beta>m_{e} / m_{i}\right)$ currents carried by fast $\left(\left(v_{b} / c_{s}\right)>\right.$ $\left.\left(R \rho_{s}\right)\left(2 \beta L_{\|}^{2}\right)\right)$, large $\left(\left(\delta_{b} / \rho_{s}\right)>2 \Lambda L_{\|} / R\right)$ filaments can enforce the effective gravity by a factor of $\left(1+\tau_{i}+\left(\omega_{A} / \omega_{B}\right)^{2}\right)$, where $\left(\omega_{A} / \omega_{B}\right)^{2} \approx R^{2} /\left(4 \beta L_{\|}^{2}\right)$ is the squared ratio of the normalized Alfvén frequency to the normalized effective gravity forcing.

In future work also effects of the parallel ion velocity and the sheath conditions as well as the impact of the ion-diamagnetic nonlinearity have to be investigated in more detail, and comparisions with simulations are aimed. 
[1] T. A. Carter, Phys. Plasmas 13, 010701 (2006).

[2] T. Windisch, O. Grulke, and T. Klinger, Phys. Plasmas 13, 122303 (2006).

[3] G. Y. Antar, J. H. Yu, and G. R. Tynan, Phys. Plasmas 14, 022301 (2007).

[4] C. Theiler, I. Furno, P. Ricci, A. Fasoli, B. Labit, S. H. Müller, and G. Plyushev, Phys. Rev. Lett. 103, 065001 (2009).

[5] T. Happel, F. Greiner, N. Mahdizadeh, B. Nold, M. Ramisch, and U. Stroth, Phys. Rev. Lett. 102, 255001 (2009).

[6] S. H. Müller, C. Theiler, A. Fasoli, I. Furno, B. Labit, G. R. Tynan, M. Xu, Z. Yan, and J. H. Yu, Plasma Phys. \& Controlled Fusion 51, 055020 (2009).

[7] I. Furno, M. Spolaore, C. Theiler, V. Vianello, R. Cavazzana, and A. Fasoli, Phys. Rev. Lett. 106, 245001 (2011).

[8] G. Fuchert, G. Birkenmeier, B. Nold, M. Ramisch, and U. Stroth, Plasma Phys. \& Controlled Fusion p. submitted (2013).

[9] M. Kocan, F. P. Gennrich, A. Kendl, H. W. Müller, and ASDEX Upgrade Team, Plasma Phys. \& Controlled Fusion 54, 085009 (2012).

[10] D. A. D'Ippolito, J. R. Myra, and S. J. Zweben, Physics of Plasmas 18, 060501 (2011).

[11] D. Jovanovic, P. K. Shukla, and F. Pegorano, Phys. Plasmas 15, 112305 (2008).

[12] N. Bisai, R. Singh, and P. K. Kaw, Phys. Plasmas 19, 052509 (2012).

[13] N. Bisai and P. K. Kaw, Phys. Plasmas 20, 042509 (2013).

[14] D. A. D’Ippolito, J. R. Myra, and S. I. Krasheninnikov, Physics of Plasmas 9, 222 (2002).

[15] S. Krasheninnikov, Phys. Lett. A 283, 368 (2001).

[16] S. I. Krasheninnikov, D. A. D’Ippolito, and J. R. Myra, J. Plasma Phys. 74, 679 (2008).

[17] W. Fundamenski, W. Sailer, and JET EFDA contributors, Plasma Phys. \& Controlled Fusion 46, 233 (2004).

[18] S. Krasheninnikov and A. Smolyakov, Phys. Plasmas 15, 055909 (2008).

[19] O. E. Garcia, N. H. Bian, and W. Fundamenski, Phys. Plasmas 13, 082309 (2006).

[20] R. Kube and O. E. Garcia, Phys. Plasmas 18, 102314 (2011).

[21] B. D. Scott, Contrib. Plas. Phys. 39, 171 (1998).

[22] B. Scott, Plasma Phys. \& Controlled Fusion 40, 823 (1998). 
[23] B. D. Scott, New J. Phys. 7, 92 (2005).

[24] B. D. Scott, Phys. Plasmas 14, 102318 (2007).

[25] A. Pakyari and V. P. Pavlenko, Phys. Plasmas 3, 3628 (1996).

[26] B. D. Scott, Phys. Plasmas 12, 062314 (2005).

[27] J. R. Angus, M. V. Umansky, and S. I. Krasheninnikov, Phys. Rev. Lett. 108, 215002 (2012).

[28] F. Militello and W. Fundamenski, Plasma Phys. \& Controlled Fusion 53, 095002 (2011).

[29] J. R. Myra, D. A. Russell, and D. A. D'Ippolito, Physics of Plasmas 13, 112502 (2006).

[30] A. Hasegawa and M. Wakatani, Phys. Rev. Lett. 50, 682 (1983).

[31] A. I. Smolyakov, J. Can. Phys. 76, 1998 (1998).

[32] R. Kube, O. E. Garcia, B. LaBombard, J. L. Terry, and S. J. Zweben, J. Nucl. Mater. p. in press (2013).

[33] N. Vianello, V. Naulin, R. Schrittwieser, H. W. Müller, M. Zuin, C. Ionita, J. J. Rasmussen, F. Mehlmann, V. rohde, R. Cavazzana, et al., Phys. Rev. Lett. 106, 125002 (2011).

[34] H. W. Müller, R. Dux, M. Kaufmann, P. T. Lang, A. Lorenz, M. Maraschek, V. Mertens, J. Neuhauser, and ASDEX Upgrade Team, Nucl. Fusion 42, 301 (2002).

[35] A. Schmid, A. Herrmann, H. W. Müller, and the ASDEX Upgrade Team, Plasma Phys. \& Controlled Fusion 50, 045007 (2008). 